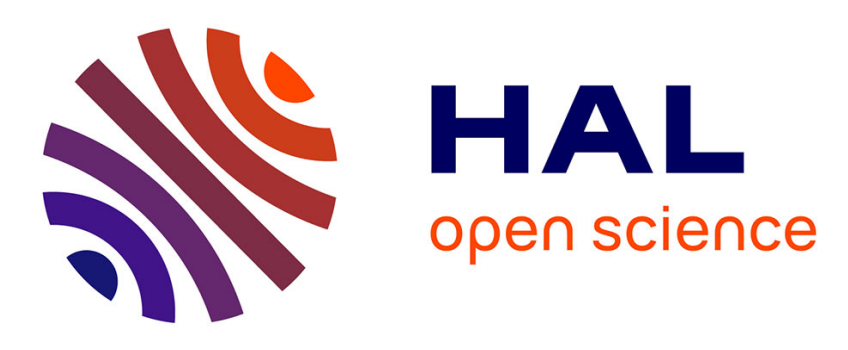

\title{
Anisotropic thermal expansion of black phosphorus from nanoscale dynamics of Phosphorene layers
}

Laura Henry, Volodymyr Svitlyk, Mohamed Mezouar, David Sifré, Gaston Garbarino, Matteo Ceppatelli, Manuel Serrano-Ruiz, Maurizio Peruzzini, Frédéric Datchi

\section{To cite this version:}

Laura Henry, Volodymyr Svitlyk, Mohamed Mezouar, David Sifré, Gaston Garbarino, et al.. Anisotropic thermal expansion of black phosphorus from nanoscale dynamics of Phosphorene layers. Nanoscale, 2020, 12, pp.4491-4497. 10.1039/C9NR09218H . hal-02437256

\section{HAL Id: hal-02437256 https://hal.science/hal-02437256}

Submitted on 29 Sep 2020

HAL is a multi-disciplinary open access archive for the deposit and dissemination of scientific research documents, whether they are published or not. The documents may come from teaching and research institutions in France or abroad, or from public or private research centers.
L'archive ouverte pluridisciplinaire HAL, est destinée au dépôt et à la diffusion de documents scientifiques de niveau recherche, publiés ou non, émanant des établissements d'enseignement et de recherche français ou étrangers, des laboratoires publics ou privés. 


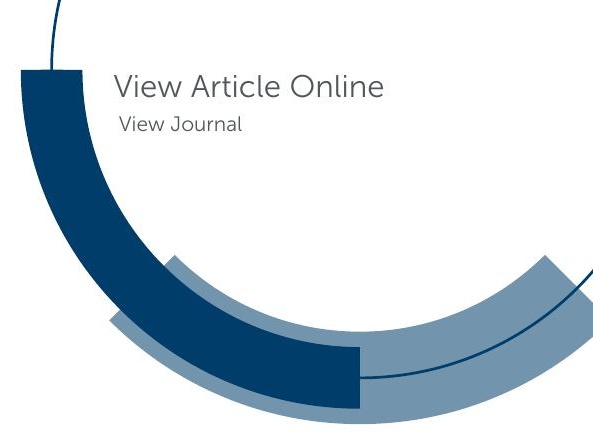

\section{Accepted Manuscript}

This article can be cited before page numbers have been issued, to do this please use: L. Henry, V. Svitlyk, M. Mezouar, D. Sifre, G. Garbarino, M. Ceppatelli, M. Serrano-Ruiz, M. Peruzzini and F. Datchi, Nanoscale, 2020, DOI: 10.1039/C9NR09218H.
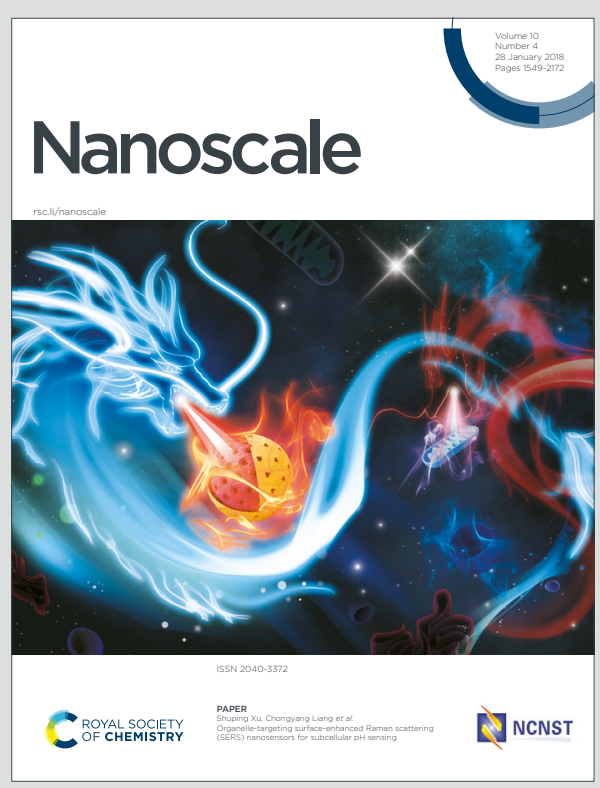

This is an Accepted Manuscript, which has been through the Royal Society of Chemistry peer review process and has been accepted for publication.

Accepted Manuscripts are published online shortly after acceptance, before technical editing, formatting and proof reading. Using this free service, authors can make their results available to the community, in citable form, before we publish the edited article. We will replace this Accepted Manuscript with the edited and formatted Advance Article as soon as it is available.

You can find more information about Accepted Manuscripts in the Information for Authors.

Please note that technical editing may introduce minor changes to the text and/or graphics, which may alter content. The journal's standard Terms \& Conditions and the Ethical guidelines still apply. In no event shall the Royal Society of Chemistry be held responsible for any errors or omissions in this Accepted Manuscript or any consequences arising from the use of any information it contains. 


\section{ARTICLE}

Received 00th January 20xx,

Accepted 00th January 20xx

DOI: $10.1039 / x 0 \times x 00000 x$

\section{Anisotropic thermal expansion of black phosphorus from nanoscale dynamics of Phosphorene layers}

Laura Henrya, Volodymyr Svitlyka, Mohamed Mezouar*a, David Sifréa, Gaston Garbarino ${ }^{a}$, Matteo Ceppatellib,c, Manuel Serrano-Ruizc ${ }^{c}$ Maurizio Peruzzinic and Frédéric Datchid

Black phosphorus (bP) is a crystalline material which can be seen as an ordered stacking of two-dimensional layers, referred to as Phosphorene. The knowledge of the linear thermal expansion coefficients (LTEC) of bP is of great interest in the field of 2D materials for a better understanding of the anistropic thermal properties and exfoliation mechanism of this material. Despite several theoretical and experimental studies important uncertainties remain in the determination of the LTEC of bP. Here, we report accurate thermal expansion measurements along the three crystallographic axes using in-situ high temperature x-ray diffraction. From the progressive reduction of the diffracted intensities with temperature we monitored the loss of the crystal structure of bP across the investigated temperature range, evidencing two thermal expansion regimes at temperature below and above $706 \mathrm{~K}$. Below $706 \mathrm{~K}$, we observe a strong out-of-plane anisotropy, while at temperatures above $706 \mathrm{~K}$ a larger thermal expansion occurs along the $a$ crystallographic direction. From our data and by taking advantage of $a b$ initio optimization, we propose a detailed anisotropic thermal expansion mechanism of bP, which leads to an inter- and intra-layer destabilization. An interpretation of it, based on the high $\mathrm{T}$ perturbation of the stabilizing $s p$ orbital mixing effect, is provided, consistently with high pressure data.

\section{Introduction}

Black phosphorus (bP) is the most stable allotrope of phosphorus at ambient conditions of pressure and temperature. ${ }^{1}$ It has been first synthesized from white phosphorus at high pressure ( $\mathrm{P}>1.2 \mathrm{GPa})$ and high temperature ( $\mathrm{T}>500 \mathrm{~K}$ ) in 1914 by P.W. Bridgman. ${ }^{2}$ bP has an orthorhombic layered structure (space group $\mathrm{Cmca}$ ) featuring strong covalent intralayer bonds between phosphorus atoms with $s p^{3}$ hybridization and relatively weak van der Waals interlayer forces which allow to obtain the single layer material called Phosphorene by means of exfoliation techniques. ${ }^{3}$ The asymmetric unit of bP contains one $\mathrm{P}$ atom located on a mirror plane ( $m$ site symmetry) and the symmetry elements of the $\mathrm{Cmca}$ group generate in total eight equivalent atoms in the unit cell (oC8 Pearson code). Due to its puckered lamellar structure bP has exceptional anisotropic mechanical properties and exhibits very contrasting directional Youngs's modulus ${ }^{4}$ and a negative Poisson's ratio. ${ }^{5}$

\footnotetext{
a. European Synchrotron Radiation Facility (ESRF), 71 Avenue des Martyrs, Grenoble France.

b. LENS, European Laboratory for Non-linear Spectroscopy, Via N. Carrara 1, I-50019 Sesto Fiorentino (FI), Italy

c. ICCOM-CNR, Institute of Chemistry of OrganoMetallic Compounds, National Research Council of Italy Via Madonna del Piano 10, I-50019 Sesto Fiorentino, Firenze (Italy)

d. Institut de Minéralogie, de Physique des Milieux Condensés et de Cosmochimie (IMPMC), Sorbonne Universités - UPMC Univ. Paris 6, CNRS UMR 7590, IRD UMR 206, MNHN, 4 place Jussieu, F-75005 Paris, France
} 
In contrast to graphene, ${ }^{6}$ which requires doping to become semiconductor, and similarly to transition metal dichalcogenide (TMD) monolayers, ${ }^{7}$ such as $\mathrm{MoS}_{2}$, Phosphorene, the single layer monoatomic of $\mathrm{P}$ atoms, whose ordered stacking builds up the orthorhombic crystal structure of bP, is inherently a 2D semiconductor with a direct band gap value of $2 \mathrm{eV}$ significantly larger than $\mathrm{bP}(0.3 \mathrm{eV})$. $^{3,8,9}$ It also exhibits a high carriers mobility $\left(\sim 1000 \mathrm{~cm}^{2} \mathrm{~V}^{-1} \mathrm{~s}^{-1}\right)$, good current on/off ratio $\left(\sim 10^{4}-10^{5}\right)$ and anisotropic in-plane properties along the zig-zag and arm-chair directions such as DC, thermal conductivity and Hall mobility, ${ }^{11}$ which appear very promising for novel applications in nanoelectronics and nanophotonics. ${ }^{10,11,12,13}$ The production of single- or multilayer Phosphorene has been successfully achieved via liquid, ${ }^{14}$ mechanical $^{3}$ or thermal thinning of bP. ${ }^{15}$ However, the exfoliation mechanism of bP still remains poorly understood and large variations of the exfoliation energy from -151 $\mathrm{meV} / \mathrm{atom}$ to $-80 \mathrm{meV} / \mathrm{atom}$ have been reported in different ab-initio simulations. ${ }^{16,17,18}$ As recently pointed out by Sansone et al., ${ }^{19}$ the discrepancy between the different theoretical calculations arises from the difficulty to accurately describe the covalent interactions and both middle- and long- range dispersive forces at the quantum mechanical level. Sansone et al. have extensively tested different ab-initio simulation approximations using the correlation between the exfoliation energy and cell volume of bP to extract an accurate exfoliation energy of -92 meV/atom evidencing a strong in-plane and outof-plane thermal expansion anisotropy. To experimentally validate this scenario, accurate thermal expansion measurements are needed but, so far, very few and limited data have been made available in the literature. ${ }^{20,21,22}$

\section{Experimental methods}

Pure highly crystalline bP was synthesized from red phosphorus as described in reference 14 . The reagents used for the synthesis were purchased from Sigma-Aldrich with the following purity grade: red phosphorus (> 99.99\%), tin (> $99.999 \%)$, gold (> 99.99\%), and $\mathrm{Snl}_{4}(99.999 \%)$. The purity of the synthesized bP crystals was verified by $x$-ray powder diffraction, Raman spectroscopy, EDX analysis and ICP-MS measurements, the latter giving a purity of 99.999+\%.

In-situ angle dispersive x-ray diffraction (XRD) experiments have been carried out at the beamline ID $27^{23}$ of the European Synchrotron Radiation Facility (Grenoble, France). A schematic of the setup is presented in Fig. 1. High and stable temperatures up to $820 \mathrm{~K}$ were generated on a large sample volume $\left(\sim 0.8 \mathrm{~mm}^{3}\right)$ placed inside a quartz capillary using a hot gas blower from the company Cyberstar positioned at the optimum distance $(\sim 5 \mathrm{~mm}$ ) from the sample. This device generates a smooth laminar flow of hot air providing a homogenous temperature distribution over a large volume of more than $10 \mathrm{~mm}^{3}$. This homogeneously heated volume is much larger than the sample $\left(\sim 1 \mathrm{~mm}^{3}\right)$ ensuring very small temperature gradients.

In order to prevent bP from reacting with the capillary and with oxygen from the hot air flow, the loading was performed in a glove bag filled with nitrogen and the powder sample was confined in a diamond cylinder of $1.0 \mathrm{~mm}$ height with $1.0 \mathrm{~mm}$ inner and $1.5 \mathrm{~mm}$ outer diameters, respectively. The Adiamond capsule was inserted in a hexagonalDBoron 0 hitfielero(ARBN) cylinder adjusted to the inner diameter of the capillary. The remaining space below the sample container was filled with non-reactive dried $\mathrm{MgO}$ powder. Finally, the capillary was sealed with epoxy in the glove bag. The temperature was regulated and determined with a precision of $\pm 1 \mathrm{~K}$ using a Eurotherm controller and a K-type thermocouple inserted inside the capillary and placed in contact with the sample assembly. The temperature was increased at a constant rate of $10 \mathrm{~K} / \mathrm{min}$ and systematically determined as the average of the temperatures measured before and after the XRD data collections. The XRD data were recorded after a temperature stabilization time of approximately 1 minute.

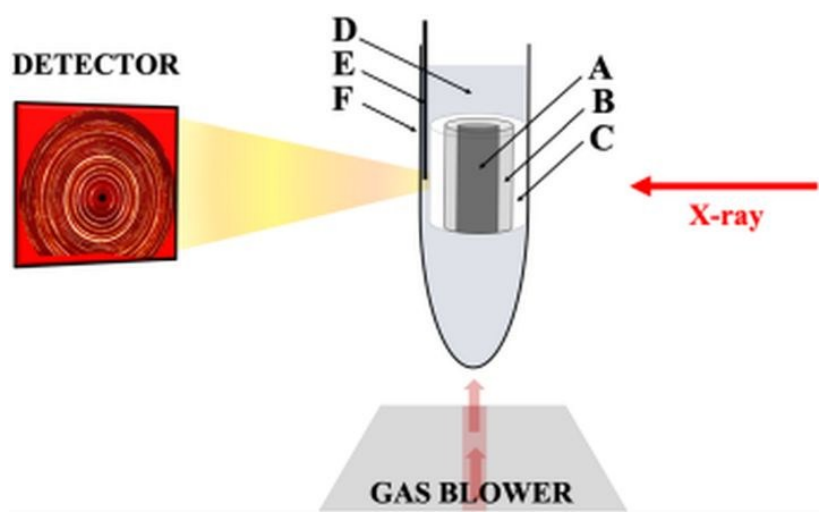

Fig. 1 Schematic of the setup: the bP powder (A) is located in a diamond cylinder (B) and encapsulated in a hexagonal boron nitride cylinder (C). The remaining space around the sample container is filled with non-reactive dried $\mathrm{MgO}$ powder (D). A K-type thermocouple $(E)$ is placed inside the quartz capillary $(F)$, in contact with the sample assembly. A gas blower is positioned below the sample at the optimum distance of $5 \mathrm{~mm}$.

We employed a highly focused $\left(3 \times 3 \mu \mathrm{m}^{2}\right.$ at FWHM), high energy $(33.17 \mathrm{keV})$ monochromatic $\mathrm{x}$-ray beam to in-situ probe the structure of solid bP. The two-dimensional diffraction patterns were recorded in transmission geometry on a MAR345 image plate detector. The sample to detector distance, detector tilt angles and beam center were accurately determined using a cerium oxide powder as standard. The two-dimensional XRD images were integrated using the PyFAI software $^{24}$ as implemented in the DIOPTAS ${ }^{25}$ suite. The program Fullprof ${ }^{26}$ was then used to refine the unit-cell parameters and volume by Le Bail extraction of d-spacings using a pseudo-Voigt peak shape function. The Le Bail refinement of bP at ambient temperature is shown in Fig. 2. We have excluded the $2 \theta$ region between 6 and $7^{\circ}$ due to the overlap of the (021) reflection of bP and (002) reflection of hBN which leads to a broad and asymmetric peak. The resulting unit-cell parameters and volume that are in excellent agreement with previous works ${ }^{20,27}$ are listed in Table 1. 


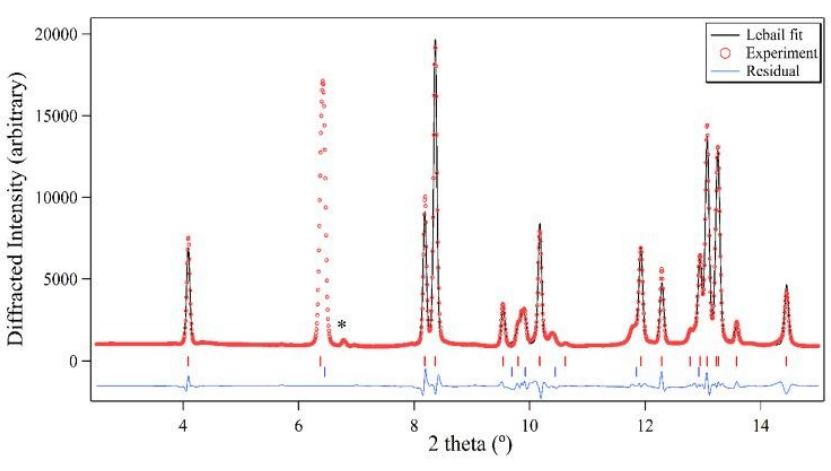

Fig. 2 Integrated diffracted intensity of powder of bP at ambient pressure and $298 \mathrm{~K}$ (open red circles), Le Bail fitting of bP (red ticks) and hBN (blue ticks) using the Fullprof software (black line) and residual between the experimental data and calculated profile (lower blue trace). A good R-factor of 3.14\% was obtained (see text for the absence of fit in the 2theta region between 6 and 7 degree). 16 phosphorus diffraction peaks were employed for the LeBail refinement of the lattice constants: the (020), (040), (111), (041), (002), (131), (022), (112), (060), (042), (200), (151), (061), (132), (220) and (221) reflections. The reflection at $6.7^{\circ}$ indicated by an asterisk is likely coming from the beamline collimating system located downstream of the sample.

\begin{tabular}{|l|l|l|l|l|l|}
\hline $\mathrm{a}(\AA)$ & $\mathrm{b}(\AA \AA)$ & $\mathrm{c}(\AA)$ & $\mathrm{V}_{0}\left(\AA^{3}\right)$ & $\lambda(\AA)$ & Ref. \\
\hline $3.3154(1)$ & $10.4771(3)$ & $4.3765(2)$ & $152.021(9)$ & 0.3738 & This work \\
\hline $3.318(6)$ & $10.47(3)$ & $4.373(9)$ & $152.00(10)$ & 0.6199 & 20 \\
\hline $3.3136(5)$ & $10.478(1)$ & $4.3763(5)$ & $151.94(3)$ & 2.0701 & 27 \\
\hline
\end{tabular}

Table 1. Unit-cell parameters $a, b$ and $c$ and unit cell volumes of bP at room temperature from this work and references 20 and 27 . The corresponding $\mathrm{x}$-ray beam wavelengths are also listed.

\section{Results and discussion}

The temperature evolution of the XRD patterns and intensities of the (020) and (111) reflections of bP are presented in Fig. 3a and $3 \mathrm{~b}$. We observed a progressive reduction of the diffraction peaks intensities at temperatures higher than $685 \mathrm{~K}$ and over a large T domain of $\sim 140 \mathrm{~K}$. At T 780 K, the XRD signal of bP completely vanished. We therefore confirm the observation by Luo et al., ${ }^{15}$ who, using Raman scattering techniques, demonstrated that bP starts to decompose at $\mathrm{T}<600 \mathrm{~K}$ with a temperature dependent process and reported slow decomposition kinetics (i.e. the speed at which bP material is removed) of around $0.18 \mathrm{~nm} \cdot \mathrm{min}^{-1}$ at $500 \mathrm{~K}$ and $1.15 \mathrm{~nm} \cdot \mathrm{min}^{-1}$ at $550 \mathrm{~K}$. The higher onset of the decomposition temperature ( $\mathrm{T}$ 685 K) observed in this work may be due to the difference in the employed characterization techniques and slow decomposition kinetics as compared to the timescale of the XRD measurements. Indeed, Raman scattering is sensitive to the surface while XRD probes the sample volume and requires a larger amount of extracted material before a significant reduction of the XRD signal can be observed. This provided a temperature-time window to determine the thermal evolution of bP beyond $685 \mathrm{~K}$ up to $780 \mathrm{~K}$. At higher $\mathrm{T}$, the sample was fully consumed and the XRD signal vanished. It is also worth noting that no traces of other phases surchlascthecpreviously reported red phosphorus ${ }^{28}$ have been observed in the entire investigated temperature domain.
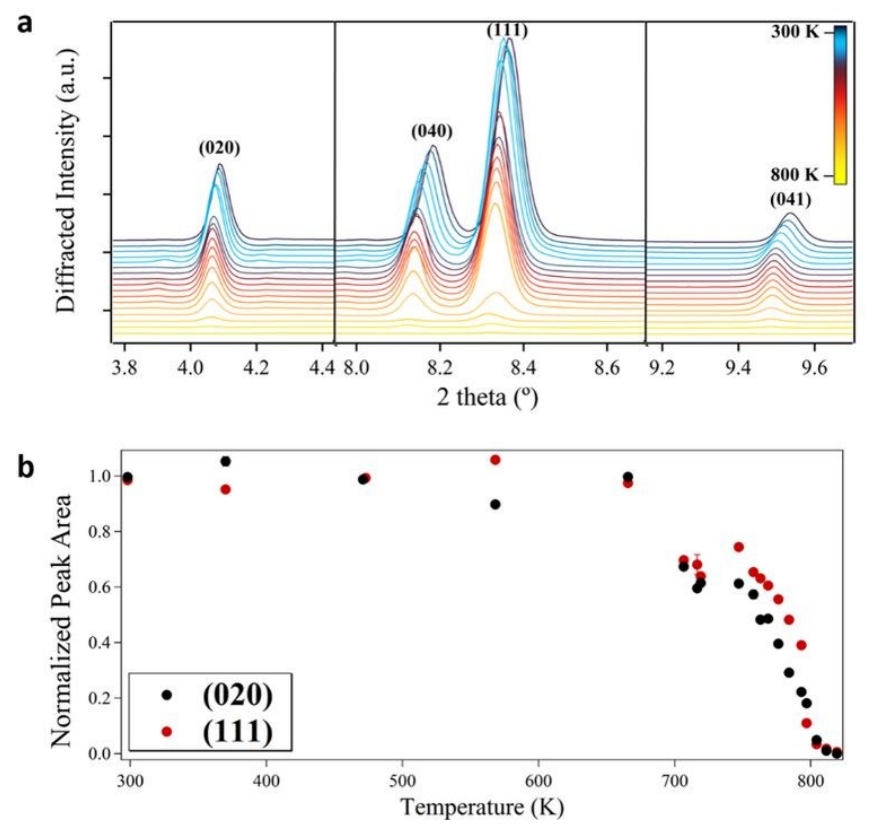

Fig. 3 (a) X-ray diffraction patterns of bP at all temperatures in the $300 \mathrm{~K}-820 \mathrm{~K}$ range for the selected (020), (040), (111) and (041) reflections. (b) Normalized peak area as a function of temperature for the (020) and (111) reflections. Above $\sim 685 \mathrm{~K}$, a sudden peak intensity drop of $30 \%$ is observed, followed by a progressive reduction to zero at $\sim 820 \mathrm{~K}$.

The temperature dependence of the unit-cell parameters and volume obtained from Le Bail fitting are presented in Fig. 4 and the resulting linear and volumetric thermal expansion coefficients are listed in Table 2. Two distinct thermal expansion regimes are observed at temperatures below and above $706 \mathrm{~K}$. Below $706 \mathrm{~K}$, a volumetric thermal expansion coefficient $\alpha_{v}$ of $24.8 \cdot 10^{-6} \mathrm{~K}^{-1}$ is obtained. This value is in good agreement with the one obtained by Faber et al. ${ }^{22}\left(\alpha_{\mathrm{v}}=22 \cdot 10^{-6}\right.$ $\mathrm{K}^{-1}$ ) using in-situ powder XRD. However, this work was cited as "private communication" in reference 19 and, to our knowledge, never published so far. This $\alpha_{v}$ value is also in close agreement with the one obtained by Sansone et al. ${ }^{19}\left(\alpha_{v}=\right.$ $20.10^{-6} \mathrm{~K}^{-1}$ ) using ab-initio computer simulations, but strongly deviates from the values reported by Keyes et al. ${ }^{21}$ and Riedner et al. $^{20}$ which, however, are both affected by large uncertainties due to the few collected data points. 

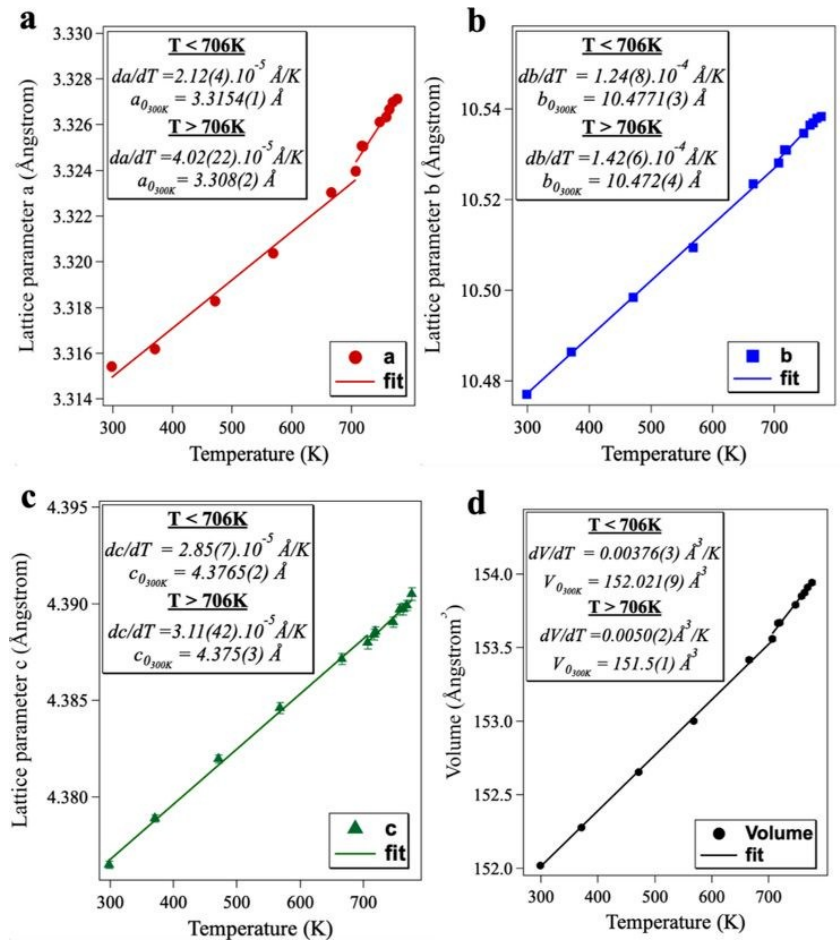

Fig. 4 Temperature dependence of the unit cell parameters $a(a), b$ (b), $c$ (c) and volume $V$ (d). The slope and intercept of the linear fits in the two temperature regimes are indicated in the insert panels.

Accurate linear thermal expansion coefficients (LTEC) $\alpha_{a}=6.4 \cdot 10^{-6} \mathrm{~K}^{-1}, \alpha_{b}=11.8 \cdot 10^{-6} \mathrm{~K}^{-1}$ and $\alpha_{c}=6.5 \cdot 10^{-6} \mathrm{~K}^{-1}$ were obtained from the corresponding linear fits of the temperature dependent unit-cell parameters along the three crystallographic directions $a, b$, and $c$. We observe a larger LTEC along the $b$ stacking direction dominated by weak van der Waals interactions (Fig. 6). This result is in qualitative agreement with the LTECs reported by Faber et al. ${ }^{22}$ and Sansone et al. ${ }^{19}$, which are indeed larger in the $b$ direction (Table 2). However, in contrast with the DFT calculations ${ }^{19}$, below $706 \mathrm{~K}$ we do not observe any in-plane anisotropy as the coefficients $\alpha_{a}$ and $\alpha_{c}$ are very close. It is worth to remark the accuracy and precision of the data presented in this study in comparison with the dispersion of the LTECs obtained from the previous powder and single-crystal XRD studies (Riedner et $a l^{20}$, and Keyes ${ }^{21}$ ) where, as already mentioned, the collection of few high-temperature data points led to poor precision and accuracy in determination of the LTECs.

\begin{tabular}{|c|c|c|c|c|c|c|}
\hline Technique & $\begin{array}{c}\alpha_{a} \\
\left(10^{-6} \mathrm{~K}^{-1}\right)\end{array}$ & $\begin{array}{c}\alpha_{b} \\
\left(10^{-6 K^{-1}}\right)\end{array}$ & $\begin{array}{c}\alpha_{\mathrm{c}} \\
\left(10^{-6 \mathrm{~K}^{-1}}\right)\end{array}$ & $\begin{array}{c}\alpha_{v} \\
\left(10^{-6} K^{-1}\right) \\
\text { DOI }\end{array}$ & $\begin{array}{c}\operatorname{Tr}(\mathbf{K}) \\
\text { View } \\
10.1039 / \mathrm{C}\end{array}$ & \begin{tabular}{|l|}
\multicolumn{1}{|c|}{ Ref. } \\
Article Online \\
9NR09218H
\end{tabular} \\
\hline Powder XRD & $6.4(1)$ & $11.8(1)$ & $6.5(2)$ & $24.8(2)$ & $300-706$ & This work \\
\hline Powder XRD & $12.2(7)$ & $13.3(6)$ & $7(1)$ & $33(1)$ & 706-780 & This work \\
\hline Single crystal & $53(5)$ & $10(2)$ & $0(5)$ & $63(12)$ & $300-475$ & [20] \\
\hline Powder XRD & $8(5)$ & $11(2)$ & $5(5)$ & $22(12)$ & $300-575$ & [22] \\
\hline Powder XRD & $22(2)$ & $38(4)$ & 39 (4) & 99 (10) & $300-700$ & [21] \\
\hline DFT & 1 & 11 & 8 & 20 & $300-600$ & [19] \\
\hline
\end{tabular}

Table 2. Volumetric and linear thermal expansion coefficients from this work and the literature.

For temperatures higher than $706 \mathrm{~K}$, we observed a drastic change in the thermal response of bP. In particular, the LTEC along the $a$ crystallographic direction strongly increases by $90 \%$ compared to $11 \%$ and to $7 \%$ respectively along the $b$ and $c$ directions. This clearly indicates an anisotropic in-plane lattice destabilization of bP above this temperature threshold.

To further exploit the XRD data, we propose a lattice destabilization mechanism which could ultimately lead to bP decomposition. It is based on the temperature evolution of the structural parameters of bP. For this purpose, we have performed an ab-initio optimization of the atomic coordinates $y$ and $z$, which represent the sole degrees of atomic freedom of orthorhombic bP, as a function of temperature. These parameters could not be directly determined by Rietveld refinement due to the presence of texture in the studied powdered sample. The optimization was performed using the Wien2k 18.2 code $^{29}$ using the PBE-GGA exchange correlation potential $^{30}$ for a k-mesh of 968 points in the Brillouin zone (BZ) (144 k-points in the irreducible region of the BZ). For each specific temperature point the lattice parameters obtained from the corresponding $x$-ray diffraction data were used without any volume optimization. As concluded from the optimization procedure and shown in Fig. 5, the $y$ coordinate steadily decreases from 0.1028 to 0.1021 while the $z$ coordinate increases from 0.0792 to 0.0798 in the $300-780 \mathrm{~K}$ temperature range. The resulting absolute shifts are 0.007 and $0.003 \AA$ along the $b$ and $c$ directions, respectively.

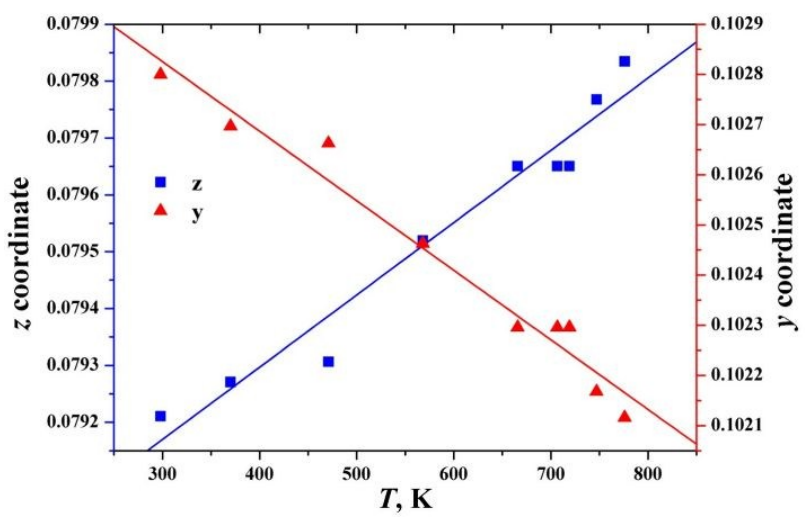

Fig. 5 Evolution of $z$ and $y$ atomic coordinates of bP as a function of temperature. 
While the absolute changes are moderate, a clear tendency towards a $T$-induced structural instability is observed. Indeed, the reduction of the $y$ coordinate induces a separation between the adjacent corrugated $\mathrm{P}$ layers along the $b$ direction (Fig. 6, middle drawing, blue arrows), while the increase of the $z$ coordinate generates antiparallel movements of $P$ atoms in the ac plane (Fig. 6, middle drawing, red arrows).

Relevant intra- and interlayer P-P distances calculated using the experimental lattice parameters and the optimized atomic positions at $300 \mathrm{~K}$ and $800 \mathrm{~K}$ are represented in Fig. 6 and listed in Table 3. From their temperature variation, we notice that the planar intralayer distances increase of $\sim 0.34 \%$ along $a$ (P1-P2 distance in Fig. 6) and $0.17 \%$ along the $a-c$ direction (P1P3 distance). More interestingly, the layer thickness (P3-P4 distance) reduces by $0.05 \%$ and the interlayer distances (P5-P3 and $\mathrm{P} 5-\mathrm{P} 1$ distances) increase by $\sim 1 \%$ resulting in an overall flattening and separation of the Phosphorene layers. This flattening is also evidenced in the temperature evolution of characteristic angles (Table 4). These changes would induce a reduction of the interlayer van der Waals interactions preluding to decomposition or exfoliation.

\begin{tabular}{|l|c|c|c|}
\hline & $\begin{array}{c}\text { Distances }(\AA) \\
\text { at T=300 K }\end{array}$ & $\begin{array}{c}\text { Distances }(\AA) \\
\text { at T=800 K }\end{array}$ & Variation \\
\hline $\begin{array}{l}\text { P1-P2 } \\
\text { (intralayer, planar along a axis) }\end{array}$ & 3.3154 & 3.327 & $+0.34 \%$ \\
\hline $\begin{array}{l}\text { P1-P3 } \\
\text { (intralayer, planar along a-c axis) }\end{array}$ & 2.2321 & 2.2359 & $+0.17 \%$ \\
\hline $\begin{array}{l}\text { P3-P4 } \\
\text { (intralayer, layer thickness) }\end{array}$ & 2.2648 & 2.2636 & $-0.05 \%$ \\
\hline $\begin{array}{l}\text { P5-P3 } \\
\text { (interlayer, layer separation) }\end{array}$ & 3.5678 & 3.6027 & $+0.98 \%$ \\
\hline $\begin{array}{l}\text { P5-P1 } \\
\text { (interlayer, layer separation) }\end{array}$ & 3.7800 & 3.8129 & $+0.87 \%$ \\
\hline
\end{tabular}

Table 3. Intra and interlayer distances in bP at $300 \mathrm{~K}$ and $800 \mathrm{~K}$ obtained from the experimental lattice parameters and optimized atomic positions. The different atomic positions (P1, P2, P3, P4 and P5) are represented in Fig. 6.

\begin{tabular}{|l|l|l|}
\hline T, K & $\begin{array}{l}\text { P1-P6-P2 } \\
\text { (degrees) }\end{array}$ & $\begin{array}{l}\text { P1-P6-P7 } \\
\text { (degrees) }\end{array}$ \\
\hline 298 & 95.91 & 101.84 \\
\hline 370 & 95.91 & 101.86 \\
\hline 471 & 95.92 & 101.86 \\
\hline 568 & 96.00 & 101.90 \\
\hline 666 & 96.05 & 101.92 \\
\hline 707 & 96.06 & 101.91 \\
\hline
\end{tabular}

Table 4. Temperature evolution of characteristic angles. The labels P1, P2, P6, P7 refer to the atoms represented in Fig. 6.

From an electronic point of view, the loss of the pseudotetrahedral local symmetry at the Phosphorus sites, associated to the flattening of the layers, correspond to a weakening in the valence shell orbital hybridization and to a consequent decrease of the stabilizing $s p^{3}$ orbital mixing, consistently with the significant increase of $\alpha_{a}$ observed above $706 \mathrm{~K}$. The originating instability is here proposed to be at the origin of the decomposition of the Phosphorene layers. This interpretation is in agreement with the work of Kumar et al. ${ }^{31}$ in which the thermal decomposition of few layer Phosphorene, studied by STM microscopy, is reported to proceed along the "zig-zag" $(a)$ direction with the formation of crater-like holes. This is consistent with the observed higher LTEC along this direction, particularly above $706 \mathrm{~K}$ and with the loss of the layers stability due to their flatteningl: 1 Remarkablik, 92tble temperature ranges of structural destabilization observed by Kumar et al. and the one reported here are in good agreement. In addition, the proposed decomposition process is compatible with the stabilizing effect proposed by Seo and Hoffmann ${ }^{32}$ for the orthorhombic structure of bP. Phosphorus is indeed the only element of group $V$ exhibiting the orthorhombic structure (A17) at ambient pressure and the one among pnictogens, where the $s p$ orbital mixing is stronger. As and $\mathrm{Sb}$, the next higher $\mathrm{Z}$ element in the group, where the $s p$ orbital mixing is increasingly weaker, adopt instead a rhombohedral layered structure (A7) at ambient conditions, which is observed in $\mathrm{P}$ only above $4.8 \mathrm{GPa}$. The key role played by the $s p$ orbital mixing in stabilizing the layered structures of Phosphorus has been recently confirmed by the observation of the existence of pseudo simple-cubic ( $p$-sc) structure at high pressure. ${ }^{33,34}$

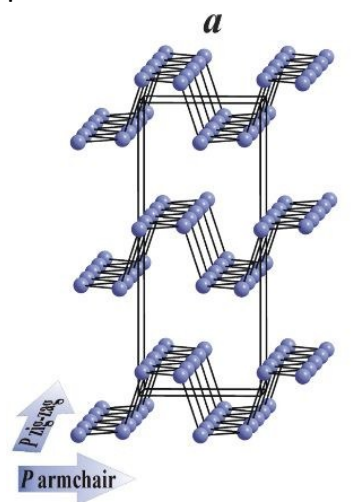

$b$

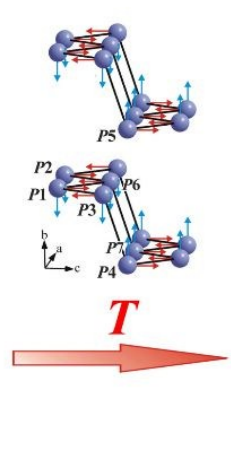

c

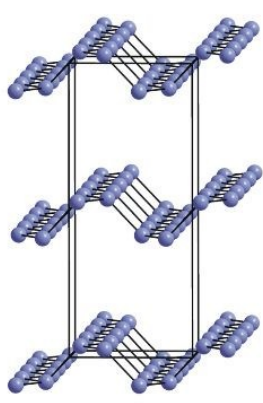

Fig. 6 (a) Crystal structure of bP indicating the "zig-zag" and armchair directions, (b) Temperature induced in-plane atomic movements as deduced from thermal expansion, (c) flattening and separation of the Phosphorene layers.

The proposed mechanism suggests that temperature induces strong structural instabilities in $\mathrm{bP}$, as concluded from the experimental and ab-initio computational data. The principal destabilization factors are the weakening of the interplanar interactions along the $b$ direction and a change in the character of the atomic interactions within the $a c$ planes corresponding to a flattening of the Phosphorene layers and an elongation along the $a$ direction.

\section{Conclusions}

We confirm from in-situ powder XRD that bP decomposes at high temperature. In contrast with a previous report ${ }^{28}$, we did not observe any intermediate phases up to the maximum investigated temperature. We have accurately determined the volumetric and linear thermal expansion coefficients and evidenced the existence of two thermal expansion regimes. At temperatures below $706 \mathrm{~K}$, we found that bP expands anisotropically mainly along the $b$ crystallographic direction, corresponding to an interlayer separation. Above this temperature, a dramatic increase of the LTEC, observed along the $a$ direction, leads to an in-plane destabilization of the 2D 
lattice of the layers, which flatten with temperature. The stabilization of the bP structure originating from the $s p$ orbital mixing is thus reduced and decomposition of bP takes place. The proposed mechanism provides a fundamental physicochemical insight about the high temperature stability of the Phosphorene layers, with potential applications to design new thermal exfoliation strategies for the synthesis of Phosphorene based materials.

\section{Conflicts of interest}

There are no conflicts to declare.

\section{Acknowledgements}

Thanks are expressed to EC through the European Research Council (ERC) for funding the project PHOSFUN "Phosphorene functionalization: a new platform for advanced multifunctional materials" (Grant Agreement No. 670173) through an ERC Advanced Grant. The authors also acknowledge the European Synchrotron Radiation Facility for provision of synchrotron beamtime at the beamline ID27, the Agence Nationale de la Recherche for financial support under Grant No. ANR 13-BS040015 (MOFLEX) and the company Almax easy Lab for providing the diamond cylinders.

\section{Author contributions}

The original idea was defined by MM. The experiments were performed by $L H, G G, D S, M C, M S R$ and $M M$ with equal contributions. The data were analysed and the figures produced by LH and V.S. with contributions from all the coauthors. The manuscript was written by $\mathrm{MM}$ and $\mathrm{MC}$ with contributions from all the co-authors.

\section{References}

1 D.R. Peck, Mellor's Comprehensive Treatise on Inorganic and Theoretical Chemistry 1971, Vol. VIII, 744-754 and D. Corbridge, Phosphorus: Chemistry, Biochemistry and Technology, Sixth Edition, Taylor \& Francis, New York, 2013.

2 P. W. Bridgman, Two new modifications of phosphorus. J. Am. Chem. Soc. 1914, 36, 1344.

3 L. Li, Y. Yu, G.J. Ye, Q. Ge, X. Ou, H. Wu, D. Feng, X.H. Chen and Y. Zhang, Black phosphorus field-effect transistors, Nat. Nanotechnol. 2014, 9, 372-377.

4 L. Vaquero-Garzon, R. Frisenda, and A. Castellanos-Gomez, Anisotropic buckling of few-layer black phosphorus, Nanoscale, 2019, 11, 12080-12086.

5 Y. Du, J. Maassen, W. Wu, Z. Luo, X. Xu and P. D. Ye, Auxetic black phosphorus: A 2D material with negative Poisson's ratio, Nano Lett. 2016, 16, 6701-6708.

6 K.S. Novoselov, A. K. Geim, S. V. Morozov, D. Jiang, Y. Zhang, S. V. Dubonos, I. V. Grigorieva and A. A. Firsov, Electric field effect in atomically thin carbon films, Science, 2004, 306, 666-669.

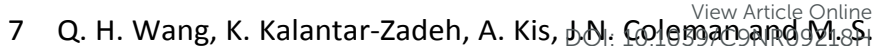
Strano, Electronics and optoelectronics of two-dimensional transition metal dichalcogenides, Nat. Nanotech. 2012, 7, 699-712.

$8 \mathrm{H}$. Asahina and A. Morita, Band structure and optical properties of black phosphorus. J. Phys. C Solid State Phys, 1984, 17, 1839-1852.

9 A. S. Rodin, A. Carvalho and A .H. Castro Neto, Strain-Induced Gap Modification in Black Phosphorus, Phys. Rev. Lett., 2014, 112, 176801.

10 X. Ling, H. Wang, S. Huang, F. Xia and M. S. Dresselhaus, The renaissance of black phosphorus, Proc. Nat. Acad. of Sci. 2015, 112, 4523-4530.

$11 \mathrm{M}$. Peruzzini, et al. A perspective on recent advances in Phosphorene functionalization and its applications in devices, Eur. J. Inorg. Chem. 2019, 11, 1476-1494.

12 H. Liu, A.T. Neal, Z. Zhu, Z. Luo, X. Xu, D. Tománek and P. D. $Y e$, Phosphorene: an unexplored $2 \mathrm{D}$ semiconductor with a high hole mobility, ACS Nano. 2014, 8, 4033-4041.

13 J. Lu, J. Yang, A. Carvalho, H. Liu, Y. Lu and C. H. Sow, Lightmatter interactions in Phosphorene, Acc. Chem. Res. 2016, 49, 1806-1815.

14 M. Serrano-Ruiz, M. Caporali, A. Ienco, V. Piazza, S. Heun and $M$. Peruzzini, The role of water in the preparation and stabilization of high-quality Phosphorene flakes. Adv. Mater. Interfaces, 2016, 3, 1500441.

15 W. Luo, R. Yang, J. Liu, Y. Zhao,W. Zhu and G. Xia, Thermal sublimation: a scalable and controllable thinning method for the fabrication of few-layer black phosphorus, Nanotechnol. 2017, 28, 285301.

16 L. Shulenburger, A. Baczewski, Z. Zhu, J. Guan and D. Tomànek, The nature of the interlayer interaction in bulk and few-layer phosphorus, Nano Lett. 2015, 15, 8170-8175.

17 D. Usvyat, L. Maschio and M. Schütz, Periodic local MP2 method employing orbital specific virtuals, J. Chem. Phys. 2015, 143, 102805.

18 G. Sansone, L. Maschio, D. Usvyat, M. Schütz and A. Karttunen, Toward an accurate estimate of the exfoliation energy of black phosphorus: A periodic quantum chemical approach, J. Phys. Chem. Lett., 2016, 7, 131-136.

19 G. Sansone, A. J. Karttunen, D. Usvyat, M. Schütz, J. G. Brandenburg and L. Maschio, On the exfoliation and anisotropic thermal expansion of black phosphorus, Chem. Commun. 2018, 54, 9793.

20 R. Riedner, S. R. Srinivasa, L. Cartz, T. G. Worlton, R. Klinger and R. Beyerlein, Anisotropic thermal expansion and compressibility of black phosphorus, AIP Conf. Proc., 1974, 820.

21 R. W. Keyes, The electrical properties of black phosphorus, Phys. Rev., 1953, 92, 580

22 In ref. 20 this work is reported as "Private Communication" by J. Faber. To our knowledge, this study was not published. 
23 M. Mezouar, et al., Development of a new state-of-the-art beamline optimized for monochromatic single crystal and powder X-ray diffraction under extreme conditions at the ESRF, J. of Synch. Rad. 2005, 12, 659-664.

24 G. Ashiotis, A. Deschildre, Z. Nawaz, J. P. Wright, D. Karkoulis, F.E. Picca and J. Kieffer, The fast azimuthal integration Python library: pyFAI, J. Appl. Cryst. 2015, 48, 510-519.

25 C. Prescher and V. B. Prakapenka, DIOPTAS: a program for reduction of two-dimensional X-ray diffraction data and data exploration, High Press. Res. 2015, 35, 223-230.

26 M. Rodriguez-Carvajal. Recent advances in magnetic structure determination by neutron powder diffraction, Physica B, 1993, 192, 55-69.

27 A. Brown and S. Rundqvist, Refinement of the crystal structure of black phosphorus, Acta Cryst., 1965, 19, 684685.

28 V. V. Brazhkin and A. J. Zerr, Relative stability of red and black phosphorus at P<1 GPa, J. Mat. Sciences, 1992, 27, 2677-2681.

29 P. Blaha, K. Schwarz, G. K. H. Madsen, D. Kvasnicka, J. Luitz, R. Laskowski, F. Tran and L. D. Marks, WIEN2k, An Augmented Plane Wave + Local Orbitals Program for calculating Crystal Properties (Karlheinz Schwarz, Techn. Universitat Wien, Austria), 2018, ISBN 3-9501031-1-2.

30 J. P. Perdew, K. Burke, M. Ernzerhof, Generalized gradient approximation made simple, Phys. Rev. Lett., 1996, 77, 3865.

31 A. Kumar, F. Telesio, S. Forti, A. Al-Temimy, C. Coletti, M. Serrano-Ruiz, M. Caporali, M. Peruzzini, F. Beltram aND S. Heun, STM study of exfoliated few layer black phosphorus annealed in ultrahigh vacuum, 2D Mater., 2019, 6, 015005.

32 D. -K. Seo and R. Hoffmann, What determines the structures of the group 15 elements? J. Solid State Chem. 1999, 147, 26 -37 .

33 D. Scelta, A. Baldassarre, M. Serrano-Ruiz, K. Dziubek, A. B. Cairns, M. Peruzzini, R. Bini and M. Ceppatelli, Interlayer bond formation in black phosphorus at high pressure, Angew. Chem. Int. Ed., 2017, 56, 14135-14140.

34 D. Scelta, A. Baldassarre, M. Serrano-Ruiz, K. Dziubek, A. B. Cairns, M. Peruzzini, R. Bini, M. Ceppatelli, The p-sc structure in phosphorus: bringing order to the high pressure phases of group 15 elements, Chem. Commun. 2018, 54, 10554. 
Nanoscale dynamics of Phosphorene layers

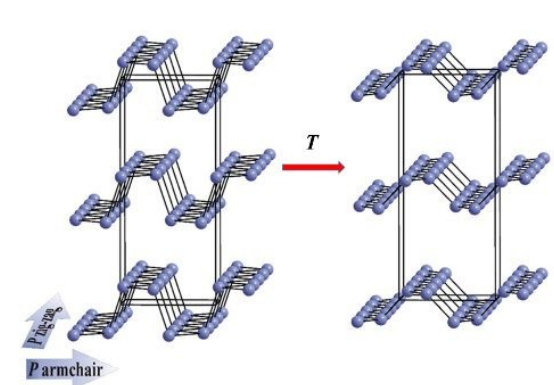

